\title{
ISQUEMIA CEREBRAL SILENTE PERIOPERATORIA EN PACIENTES CON FIBRILACIÓN AURICULAR
}

\author{
PERIOPERATIVE SILENT CEREBRAL ISCHEMIA IN PATIENTS WITH ATRIAL \\ FIBRILLATION
}

\author{
ALEJANDRO E. CONTRERAS ${ }^{1}$, FEDERICO ROCA ${ }^{2}$, JOSE I. REVIGLIONO ${ }^{3}$, FLORENCIA BECERRA ${ }^{4}$, TERESITA CORNAVACA ${ }^{4}$, RICARDO A. \\ ALBERTINI ${ }^{3}$, ALDO H. TABARES ${ }^{5}$
}

\section{RESUMEN}

Introducción. Frecuentemente en la práctica clínica se plantea la necesidad de revertir la anticoagulación con antagonistas de la vitamina K en pacientes con fibrilación auricular (FA) previo a la realización de procedimientos invasivos. Trabajos científicos recientemente publicados sugieren que es segura la suspensión del tratamiento anticoagulante sin necesidad de terapia sustitutiva con heparina. Objetivos. Determinar si los pacientes con FA decon bajo riesgo tromboembólico presentaron eventos cerebrales isquémicos silentes durante intervenciones invasivas luego de la suspensión de la anticoagulación.

Métodos. Estudio observacional, prospectivo, que se realizó mediante el seguimiento clínico de pacientes internados para procedimiento invasivos desde el año 2010 a la actualidad en el Hospital Privado Universitario de Córdoba. Resultados. Fueron evaluados 7 pacientes con FA de bajo riesgo tromboembólico, a quienes se los estudió mediante resonancia nuclear magnética cerebra pre- y posquirúrgicas (en secuencias de difusión, T2 y FLAIR). La totalidad de la muestra tenía FA, encontrándose bajo tratamiento anticoagulante con antagonistas de la vitamina $\mathrm{K}$, y tenía una mediana de $\mathrm{CHA}_{2} \mathrm{DS}_{2}$-VASC de 2 puntos (0-2 puntos, riesgo tromboembólico bajo). El día de la intervención quirúrgica el grupo de estudio presentó un RIN promedio de $1,36(1,02-1,86)$ y necesitaron para alcanzar el rango terapéutico de anticoagulación 4,86 días (4-5 días). Ninguno recibió heparinas en dosis anticoagulantes y un solo paciente recibió profilaxis. No se evidenciaron eventos neurológicos clínicamente evidentes, sin embargo en un paciente $(14,2 \%)$ se observó una imagen isquémica cerebral en el posoperatorio, sin repercusión clínica.

Conclusión. A pesar del pequeño número casos, nuestra experiencia genera la hipótesis de que algunos pacientes con FA con bajo riesgo de tromboembolismo perioperatorio podrían tener isquemia silente sin terapia anticoagulante. Un estudio futuro con mayores dimensiones teniendo en cuenta el momento de realización de las imágenes y el seguimiento a largo plazo podría dilucidar la frecuencia de la isquemia silente y sus consecuencias

Palabras clave: isquemia encefálica; anticoagulantes; tromboembolia; cirugía.

\section{ABSTRACT}

Introduction. Frequently in clinical practice the need to reverse anticoagulation with vitamin $\mathrm{K}$ antagonists in patients with atrial fibrillation (AF) prior to performing invasive procedures is considered. Recently published scientific papers su ggest that the suspension of anticoagulant therapy is safe without the need for heparin replacement therapy.

Objective. To determine whether patients with AF with low thromboembolic risk presented silent cerebral ischemic events during invasive interventions after the suspension of anticoagulation.

Methods. An observational, prospective study was carried out through the clinical follow-up of inpatients for invasive procedures from 2010 to the present at the Private University Hospital of Córdoba.

Results. Seven patients with AF of low thromboembolic risk were evaluated, who were assessed by cerebral magnetic resonance imaging before and after surgery (in diffusion, T2 and FLAIR sequences). The whole sample had AF and were anticoagulated with vitamin $\mathrm{K}$ antagonists and had a 2-point $\mathrm{CHA}_{2} \mathrm{DS}_{2}-\mathrm{VASC}$ median (0-2 points, low thromboembolic risk). On the day of the surgical intervention, the study group presented an average INR of 1.36 (1.02-1.86) and needed 4.86 days (4-5 days) to reach the therapeutic anticoagulation range. None received heparins in anticoagulant doses and only one patient received prophylaxis. No clinically evident neurological events were evident, how ever one patient (14.2\%) showed a cerebral ischemic image in the postoperative period, without clinical repercussions.

Conclusion. Despite the small number of cases, our experience generates the hypothesis that some patients with AF with a low risk of perioperative thromboembolism could have silent ischemia without anticoagulant therapy. A future study with larger dimensions taking into account the moment of performance of the images and long-term follow-up, could elucidate the frequency of silent ischemia and its consequences.

Keywords: ischemia; anticoagulants; thromboembolism; surgery.

REVISTA CONAREC 2018;33(145):184-186 | DOI:10.32407/RCON/2018145/0184-0186

\footnotetext{
1. Médico de Planta, Servicio de Cardiología.

2. Médico de Planta, Servicio de Radiología.

3. Médico de Planta, Servicio de Medicina Interna.

4. Médico Residente, Servicio de Medicina Interna.

5. Jefe de Servicio de Medicina Vascular.

Hospital Privado Universitario de Córdoba. Instituto Universitario de Ciencias Biomédicas de Córdoba. Córdoba,. Argentina.
}

$\triangle$ Correspondencia: Dr. Alejandro Contreras. Naciones Unidas 346, Córdoba, Rep. Argentina.aletreras@hotmail.com.

Los autores declaran no poseer conflictos de intereses.

Recibido: 10/11/2017 | Aceptado: 01/06/2018

\section{INTRODUCCIÓN}

En los pacientes con fibrilación auricular (FA) de bajo a moderado riesgo embolígeno en tratamiento con anticoagulantes orales (ACO) que deben ser sometidos a procedimientos invasivos, la estrategia de suspensión del anticoagulante 5 días previos al procedimiento, comparada con el tratamiento que incluye el cabalgamiento con heparinas periprocedimiento, ha demostrado tener resultados similares en cuanto a eventos tromboembólicos. Por lo cual, actualmente, se sugiere la suspensión del ACO previamente 1 .

Luego de cirugías valvulares o de ablación de FA por radiofrecuencia, la evaluación mediante resonancia magnética cerebral ha demostrado la presencia de isquemia cerebral silente posprocedimiento ${ }^{2,3}$. El objetivo del presente trabajo es determinar si los pacientes con FA de riesgo tromboembólico bajo, en quienes se recomienda la sus- 
Tabla 1. Características de la población

\begin{tabular}{|c|c|c|c|c|c|c|c|c|}
\hline & Edad (años) & Género & CHA2DS2-VASC & Cirugía & RIN previo & RM Preop. (días) & RM Postop. (días) & Isquemia silente \\
\hline 1 & 70 & Mujer & 1 & Cataratas & $1,86^{*}$ & 5 & 0 & Sí \\
\hline 2 & 50 & Varón & 0 & Artroscopia de mano & 1,15 & 6 & 10 & No \\
\hline 3 & 70 & Varón & 1 & Implantes dentarios & 1,09 & 7 & 29 & No \\
\hline 4 & 81 & Mujer & 2 & Cataratas & 1,48 & 9 & 27 & No \\
\hline 5 & 86 & Mujer & 2 & Artroscopia de mano & $1,73^{* *}$ & 1 & 56 & No \\
\hline 6 & 81 & Mujer & 2 & Reemplazo total de cadera & 1,02 & 36 & 2 & No \\
\hline 7 & 82 & Mujer & 2 & Polipectomía vesical & 1,17 & 27 & 33 & No \\
\hline
\end{tabular}

Administración de vitamina K preoperatoria vía oral: * $2 \mathrm{mg} ;{ }^{* *} 3 \mathrm{mg}$.

RIN: razón internacional normatizada. RM: resonancia magnética. Preop: preoperatorio. Postop: posoperatorio

pensión del ACO sin realizar terapia puente (bridging) con heparina, presentan eventos cerebrales isquémicos silentes al someterse a procedimientos invasivos.

\section{MATERIAL Y MÉTODOS}

Estudio observacional, prospectivo, que se realizó mediante el seguimiento clínico de pacientes internados para procedimiento invasivos desde el año 2010 a la actualidad en el Hospital Privado Universitario de Córdoba. Inicialmente se analizó cada participante mediante un protocolo confeccionado en esta institución ${ }^{4}$, el cual avala que pacientes con FA de bajo riesgo tromboembólico $\left(\mathrm{CHA}_{2} \mathrm{DS}_{2}\right.$-VASc menor o igual a 2) suspendan la medicación anticoagulante 4 a 5 días previos al procedimiento, sin realizar terapia con heparinas. Luego fueron evaluados mediante resonancia magnética (RM) de cerebro, utilizando secuencias de cortes axiales en T2, FLAIR y difusión, sagitales en T1 y coronales en T2, pre- y posoperatorias. Las imágenes fueron analizadas y comparadas por un especialista en neurorradiología. Se utilizó un resonador de 1,5 tesla. Todos los participantes firmaron un consentimiento informado.

Las variables categóricas se expresan en porcentaje y las variables continuas en promedio y desvío estándar. Para su análisis se usó el paquete estadístico InfoStat/P (Universidad Nacional de Córdoba, 2014).

\section{RESULTADOS}

Fueron evaluados 126 pacientes intervenidos en 159 procedimientos, de los cuales 82 (52\%) no recibieron terapia "puente" con enoxaparina. Se realizaron imágenes de RM cerebral en 18 casos antes del procedimiento invasivo. Posteriormente, al excluirse los pacientes que realizaron "puente" con enoxaparina y aquellos a los que no se les realizó control con RM en el posoperatorio, el tamaño de la muestra fue de 7 pacientes con RM pre- y posoperatoria, a quienes se les suspendió el ACO y no fueron tratados con enoxaparina.

El grupo en estudio estaba formado por 5 mujeres (71\%) y 2 hombres (29\%), con edad promedio de 74,2 años (50-86 años). La totalidad de la muestra tenía FA, encontrándose anticoagulados con antagonistas de la vitamina $\mathrm{K}$, con una mediana de $\mathrm{CHA}_{2} \mathrm{DS}_{2}$-VASC de 2 puntos (0-2 puntos, riesgo tromboembólico bajo). El día de la intervención quirúrgica el grupo de estudio presentó un RIN promedio de 1,36 $\left(1,02^{-1} 1,86\right)$ y necesitaron para alcanzar el rango terapéutico de anticoagulación 4,86 días

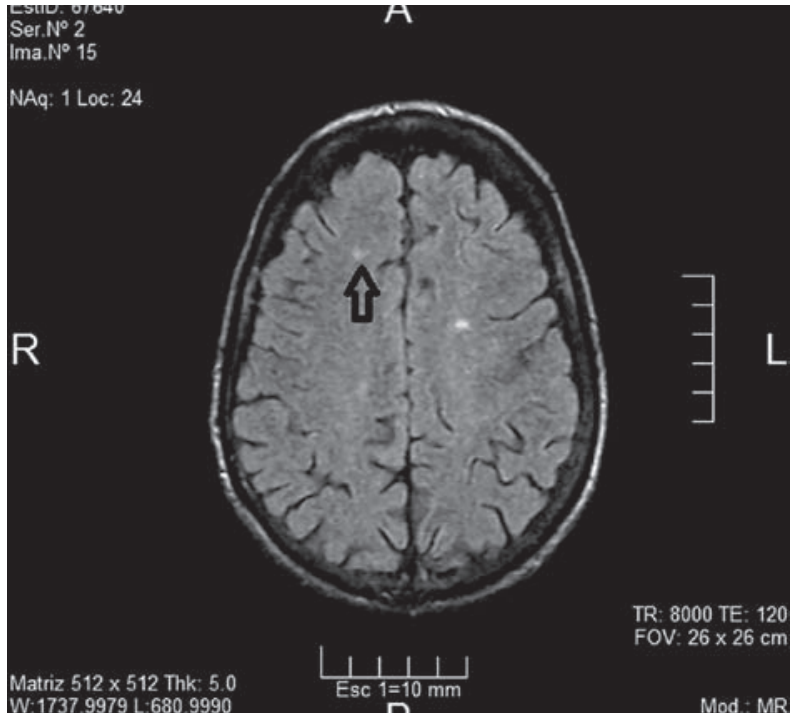

Figura 1. Resonancia magnética (FLAIR) realizada en tiempo posquirúrgico evidenciando imagen embólica subcortical frontal derecha.

(4-5 días). Ninguno recibió heparinas en dosis anticoagulantes y un solo paciente recibió profilaxis. No se evidenciaron eventos neurológicos clínicamente evidentes, sin embargo un paciente presentó una lesión isquémica silente nueva (14,2\% del total). Dicha imagen isquémica fue observada en el paciente 1 (Tabla 1), cuya RM preoperatoria se había realizado 5 días previos a la cirugía y posprocedimiento el mismo día de la cirugía. Se observó una nueva imagen puntiforme, hiperintensa en T2 y FLAIR, subcortical frontal anterior derecha, no observada en la RM preoperatoria (Figura 1)

\section{DISCUSIÓN}

El principal hallazgo de nuestro trabajo fue encontrar un caso de isquemia cerebral silente en pacientes con FA no valvular de bajo riesgo tromboembólico. Las microembolias son más frecuentes que los eventos con manifestaciones clínicas y las imágenes de difusión demuestran una incidencia de entre 10 y 40\% periprocedimiento de distintas intervenciones ${ }^{3}$. Es controvertido el significado de dicho hallazgo ya que no presentaron correlato clínico y esto se corresponde con isquemia silente sin consecuencias en otros escenarios perioperatorios ${ }^{2,5}$, sin embargo otros autores han demostrado que dichos eventos diagnostica- 
dos por RM se correlacionan con desarrollo de deterioro cognitivo y demencia en el seguimiento ${ }^{6}$, o incluso podrían ser predictores de eventos clínicos cerebrovasculares futuros?

Park et al. en un estudio de pacientes sometidos a intervenciones carotídeas $^{8}$, demostraron que la mayoría de las lesiones embólicas son de pequeño tamaño (menos de $5 \mathrm{~mm}$ de diámetro). Y el tamaño y la extensión de la lesión así como la modalidad de imagen utilizada para el diagnóstico de isquemia silente tienen suma importancia, ya que solo la secuencia de difusión en RM permite detectar lesiones isquémicas recientes, ocurridas incluso dentro de las primeras horas, permitiendo caracterizar el edema citotóxico que generan al restringir la difusión del agua en el espacio extracelular. Las lesiones preceden en varias horas a su visualización en secuencias habituales como T2 y FLAIR, y aún más para la tomografía computarizada. Sin embargo, en estudios experimentales con animales, las imágenes FLAIR demostraron correlacionarse con infartos confirmados histopatológicamente. En cambio, es incierto si las lesiones positivas en difusión, posteriormente con el tiempo, son positivas en FLAIR. Por último, existen diferencias en cuanto a la sensibilidad de la resonancia, al comparar imágenes con resonadores de 1,5 vs. 3.0 tesla9.
Nuestra experiencia tiene limitaciones, principalmente referidas al número de pacientes y al momento de realizar la RM previa y posterior a la cirugía. Hay una gran variabilidad en los tiempos en los que fueron hechas y es de notar que el único caso en que la RM posterior fue positiva se realizó el mismo día de la cirugía y fue positivo en imágenes T2 y FLAIR, por lo que probablemente el evento isquémico ocurrió antes del procedimiento. Se podrían haber perdido isquemias silentes si se hubiesen realizado las imágenes en las primeras horas o días luego de la cirugía.

\section{CONCLUSIÓN}

A pesar del pequeño número casos, nuestra experiencia genera la hipótesis de que algunos pacientes con FA de bajo riesgo de tromboembolismo perioperatorio podrían tener isquemia silente sin terapia anticoagulante. Un estudio futuro con mayores dimensiones teniendo en cuenta el momento de realización de las imágenes y el seguimiento a largo plazo, podría dilucidar la frecuencia de la isquemia silente y sus consecuencias.

\section{BIBLIOGRAFÍA}

1. Douketis JD, Spyropoulos AC, Kaatz S, Becker RC, Caprini JA, Dunn AS et al. Perioperative bridging anticoagulation in patients with atrial fibrillation. $N$ Engl J Med 2015;373(9):823-33

2. Jeon SB, Lee JW, Kim SJ, Chung CH, Kwon SU, Choi CG et al. New cerebral lesion on T2-weighted gradient-echo imaging after cardiac valve surgery. Cerebrovasc Dis 2010;30(2):194-9.

3. Gaita F, Caponi D, Pianelli M, Scaglione M, Toso E, Cesarani F et al. Radiofrequency catheter ablation of atrial fibrillation: A cause of silent thromboembolism? Magnetic resonance imaging assessment of cerebral thromboembolism in patients undergoing ablation of atrial fibrillation. Circulation 2010;122(17):1667-73.

4. Revigliono Jl, Cornavaca T, Becerra F, Albertini R, Contreras AE, Tabares A. Control perioperatorio de la anticoagulación con antagonistas de la vitamina K en cirugía electiva. Medicina (B Aires) 2014:74(5):385-90

5. Lickfett L, Hackenbroch M, Lewalter T, Selbach S, Schwab JO, Yang A et al. Cerebral diffusion-weighted magnetic resonance imaging: A tool to monitor the thrombogenicity of left atrial catheter ablation. I Cardiovasc Electrophysiol 2006; 17(1):1-7
6. Vermeer SE, Prins ND, den Heijer T, Hofman A, Koudstaal PJ, Breteler MMB. Silent brain infarcts and the risk of dementia and cognitive decline. N Engl J Med 2003;348(13):1215-22.

7. Kang DW, Lattimore SU, Latour LL, Warach S. Silent ischemic lesion recurrence on magnetic resonance imaging predicts subsequent clinical vascular events. Arch Neurol 2006;63(12):1730-3.

8. Park Kl, Chung PW, Kim YB, Moon HS, Suh BC, Yoon WT. Post-interventional microembolism: Cortical border zone is a preferential site for ischemia. Cerebrovasc Dis 2011;32(3):269-75.

9. Deneke T, Jais P, Scaglione M, Schmitt R, Di Biase L, Christopoulos G et al. Silent cerebral events/lesions related to atrial fibrillation ablation: A clinical review. J Cardiovasc Electrophysiol 2015;26(4):455-63. 